\title{
Spurious solutions for the advection-diffusion equation using wide stencils for approximating the second derivative
}

Hannes Frenander and J an Nordström

The self-archived postprint version of this journal article is available at Linköping University Institutional Repository (DiVA):

http:/ / urn.kb.se/ resolve?urn=urn:nbn:se:liu:diva-141208

N.B.: When citing this work, cite the original publication.

Frenander, H., Nordström, J ., (2017), Spurious solutions for the advection-diffusion equation using wide stencils for approximating the second derivative, Numerical Methods for Partial Differential Equations. https:// doi.org/ 10.1002/ num.22210

Original publication available at:

https:// doi.org/ 10.1002/ num.22210

Copyright: Wiley (12 months)

http:/ / eu.wiley.com/WileyCDA/ 


\title{
Spurious solutions for the advection-diffusion equation using wide stencils for approximating the second derivative
}

\author{
Hannes Frenander ${ }^{\mathrm{a}}$, Jan Nordström ${ }^{\mathrm{b}}$ \\ ${ }^{a}$ Department of Mathematics, Computational Mathematics, Linköping University, \\ SE-581 83 Linköping, Sweden (hannes.frenander@liu.se). Phone: +46-13-28 1471 \\ ${ }^{b}$ Department of Mathematics, Computational Mathematics, Linköping University, \\ SE-58183 Linköping, Sweden (jan.nordstrom@liu.se). Phone: +46-13-281459
}

\begin{abstract}
A one dimensional steady-state advection-diffusion problem using summationby-parts operators is investigated. For approximating the second derivative, a wide stencil is used, which simplifies implementation and stability proofs. However, it also introduces spurious, oscillating, modes for all mesh-sizes. We prove that the size of the spurious modes are equal to the size of the truncation error for a stable approximation and hence disappears with the convergence rate. The theoretical results are verified with numerical experiments.
\end{abstract}

Keywords: Summation-by-parts, spurious solutions, oscillating solutions.

\section{Introduction}

Finite difference methods using central difference schemes often result in spurious modes, especially if second derivatives are computed using the first derivative operator twice. These modes, which are products of the discretization of the problem, have no connection to the continuous partial differential equation, and are therefore unwanted. One way to reduce these oscillations is by constructing appropriate boundary closures $[1,2]$. Another way is to add artificial dissipation to the scheme $[3,4,5]$. The oscillations can also be reduced by using compact operators $[6,7]$ (operators with minimal stencil width). However, proving stability by energy methods and implementing fluxes when using compact operators can be cumbersome when mixed deriva- 
tives together with second order ones exist, as in the compressible NavierStokes equations. These issues vanish when using the first derivative operator twice for the second derivatives. In this paper we ask the question: are the oscillating spurious modes a real problem, or can they be ignored?

Assuming that well-posed boundary conditions and a stable numerical scheme is in place, the spurious oscillations for the steady-state advectiondiffusion problem will be analyzed. In particular, we will determine the rate with which these oscillations vanish during mesh refinement. We focus on finite difference operators with the Summation-By-Part (SBP) property, and weakly imposed boundary conditions. By using the SBP operators $[8$, 6], augmented with Simultaneous Approximation Terms (SAT) [9], a stable numerical scheme can be obtained when well-posed boundary conditions are available [7]. A comprehensive review of the SBP-SAT technique is given in $[5]$.

The rest of this paper will proceed as follows. In Section 2, well-posed boundary conditions for the advection-diffusion equation in one space dimension are determined. Next, the SBP-SAT method is applied to the equation, and stable penalty parameters are derived. In Section 3, we will prove that the rate of convergence is two for the second order accurate scheme in the steady-state limit. The results from Section 3 are generalized to higher order stencils in Section 4. In Section 5, the theoretical results are tested in numerical experiments. Finally, in Section 6, we summarize the results of this work, and draw conclusions.

\section{The advection-diffusion equation}

Consider the advection-diffusion equation in the domain $x \in[0,1]$ and $t \geq 0$

$$
\begin{aligned}
u_{t}+a u_{x} & =\epsilon u_{x x} \\
c_{1} u(0, t)-\epsilon u_{x}(0, t) & =g_{0}(t) \\
c_{2} u(1, t)-\epsilon u_{x}(1, t) & =g_{1}(t) \\
u(x, 0) & =f(x) .
\end{aligned}
$$

39 In $(1), g_{0,1}(t)$ are the boundary data, $f(x)$ is the initial data, $c_{1,2}$ are constants to be determined such that (1) becomes well-posed and $\epsilon, a>0$. 


\subsection{Well-posedness}

By multiplying (1) with $u$ and integrating over the domain, we obtain

$$
\|u\|_{t}^{2}+2\left\|u_{x}\right\|^{2}=\left[a u^{2}-2 \epsilon u u_{x}\right]_{x=0}-\left[a u^{2}-2 \epsilon u u_{x}\right]_{x=1},
$$

where $\|u\|^{2}=\int_{0}^{1} u^{2} d x$. The boundary data does not influence the wellposedness of the problem, so we let $g_{0}=g_{1}=0$ for simplicity [10]. Inserting the homogeneous boundary conditions from (1) into (2), yields

$$
\|u\|_{t}^{2}+2 \epsilon\left\|u_{x}\right\|^{2}=u^{2}(0, t)\left(a-2 c_{1}\right)-u^{2}(1, t)\left(a-2 c_{2}\right)
$$

and an energy estimate is obtained by choosing $c_{1} \geq a / 2$ and $c_{2} \leq a / 2$. With these choices of $c_{1,2}$, the solution $u$ is bounded. The energy estimate (3) automatically lead to uniqueness, and existence is guaranteed since the right number of boundary conditions are used. We summarize the results in the following proposition.

Proposition 1. Equation (1) is well-posed if $c_{1} \geq a / 2$ and $c_{2} \leq a / 2$.

In the remainder of this paper, we use $c_{1}=a, c_{2}=0$ and (1) is stated as

$$
\begin{aligned}
u_{t}+a u_{x} & =\epsilon u_{x x} \\
a u(0, t)-\epsilon u_{x}(0, t) & =g_{0}(t) \\
\epsilon u_{x}(1, t) & =g_{1}(t) \\
u(x, 0) & =f(x) .
\end{aligned}
$$

\subsection{Stability}

When discretizing (4), we use the SBP operator $D=P^{-1} Q$, where $P=$ $P^{T}>0$ and proportional to the grid spacing $h$. The matrix $Q$ satisfy the SBP property $Q+Q^{T}=\operatorname{diag}(-1,0 \ldots, 0,1)$. The operators $P$ and $Q$ are constructed such that the discrete energy estimate mimics the continuous one in (3). In this work, we consider SBP finite difference operators with diagonal $P$. The operators are of order $2 s$ in the interior and $s$ at a finite number of grid points within the boundary closures. The overall accuracy of the scheme becomes $s+1$ [11]. We henceforth denote these operators as $S B P(2 s, s)$.

We have,

$$
\begin{aligned}
v_{t}+a P^{-1} Q v & =\epsilon\left(P^{-1} Q\right) v_{x}+\alpha_{0} P^{-1}\left(a v_{0}-\epsilon\left(v_{x}\right)_{0}-g_{0}\right) e_{0} \\
& +\alpha_{1} P^{-1}\left(\epsilon\left(v_{x}\right)_{N}-g_{1}\right) e_{N} \\
v(0) & =f
\end{aligned}
$$


where the notation $v_{x}=P^{-1} Q v$ is used, i.e a wide stencil approximates the second order derivative. In $(5), v=\left(v_{0}, v_{1} \ldots, v_{N}\right)^{T}$ is the semi-discrete approximation of $u$ at each grid point. The penalty parameters $\alpha_{0,1}$ in the SAT's will be determined such that the approximation becomes stable. Also, $e_{0}=[1,0 \ldots, 0]^{T}$ and $e_{N}=[0 \ldots, 0,1]^{T}$.

By multiplying (5) with $v^{T} P$ from the left, adding the transpose of the outcome to itself and using the SBP-property of $Q$, one arrives at

(1)

$$
\begin{aligned}
\frac{d}{d t}\left(\|v\|_{P}^{2}\right)+2 \epsilon\left\|v_{x}\right\|_{P}^{2} & =v_{0}\left(a v_{0}-2 \epsilon\left(v_{x}\right)_{0}\right)-v_{N}\left(a v_{N}-2 \epsilon\left(v_{x}\right)_{N}\right) \\
& +2 \alpha_{0} v_{0}\left(a v_{0}-\epsilon\left(v_{x}\right)_{0}\right)+2 \alpha_{1} v_{N}\left(\epsilon\left(v_{x}\right)_{N}\right),
\end{aligned}
$$

where again zero boundary data is used. In (6), the norm is defined by $\|v\|_{P}^{2}=v^{T} P v$. By choosing $\alpha_{0}=\alpha_{1}=-1,(6)$ is reduced to

$$
\frac{d}{d t}\left(\|v\|_{P}^{2}\right)+2 \epsilon\left\|v_{x}\right\|_{P}^{2}=-a v_{0}^{2}-a v_{N}^{2} \leq 0 .
$$

In (7), we observe that the solution $v$ is bounded, and the numerical scheme (5) is therefore stable. Note that, due the the SBP property of $P$ and $Q$, (7) mimics (3) if $c_{1}=a$ and $c_{2}=0$. For a more detailed discussion of well-posedness and stability, the reader is referred to [5]. We summarize the results of this section in the following proposition.

Proposition 2. The semi-discrete formulation (5) is stable if $\alpha_{0}=\alpha_{1}=-1$.

\section{Spurious oscillations for the second order accurate case}

The semi-discrete form of (4) in the steady-state limit (i.e. $v_{t}=0$ ) is

$$
a P^{-1} Q v=\epsilon\left(P^{-1} Q\right) v_{x}-P^{-1}\left(a v-\epsilon\left(v_{x}\right)_{0}-g_{0}\right) e_{0}-P^{-1}\left(\epsilon\left(v_{x}\right)_{N}-g_{1}\right) e_{N} .
$$

Since we are studying a steady-state problem, the boundary data $g_{0,1}$ are constants. We start by considering the second order SBP-stencil, where the internal discrete approximation is given by

$$
a \frac{v_{i+1}-v_{i-1}}{2 h}=\frac{\epsilon}{4 h^{2}}\left(v_{i+2}-2 v_{i}+v_{i-2}\right)
$$

for $2 \leq i \leq N-2$ and grid spacing $h$. Equation (9) can be solved by making the ansatz $v_{i} \sim K^{i}$ where $K$ depends on $h$. Inserting the ansatz into (9) gives the relation

$$
\frac{K^{2}-1}{2}\left(\frac{K^{2}-1}{2}-\theta K\right) K^{i-4}=0
$$


where $\theta=a h / \epsilon$ is the so-called mesh Reynolds number.

The non-trivial solutions to (10) are

$$
\begin{aligned}
& K_{1}=1 \\
& K_{2}=\theta+\sqrt{1+\theta^{2}}=1+\theta+\frac{\theta^{2}}{2}+\mathcal{O}\left(\theta^{4}\right) \\
& K_{3}=-1 \\
& K_{4}=\theta-\sqrt{1+\theta^{2}}=-1+\theta-\frac{\theta^{2}}{2}+\mathcal{O}\left(\theta^{4}\right) .
\end{aligned}
$$

The spurious, or oscillating, modes are $K_{3,4}$, due to the negative sign. These modes have no relevance to the physical problem and introduce errors in the numerical solution.

The solution at each grid point $i$ is a weighted sum of all modes and can be written as

$$
v_{i}=\sum_{k=1}^{4} \sigma_{k} K_{k}^{i} .
$$

To determine the coefficients $\sigma_{1-4}$, we consider the boundary closures of the scheme. These conditions are,

$$
\begin{aligned}
a \theta\left(v_{1}-v_{0}\right)-a\left(v_{2}-2 v_{1}+v_{0}\right)+a \theta v_{0}-a\left(v_{1}-v_{0}\right) & =\theta g_{0}, \\
a \theta\left(v_{2}-v_{0}\right)-\frac{a}{2}\left(\frac{1}{2} v_{3}-\frac{3}{2} v_{1}+v_{0}\right) & =0, \\
a \theta\left(v_{N}-v_{N-2}\right)-\frac{a}{2}\left(\frac{1}{2} v_{N-3}-\frac{3}{2} v_{N-1}+v_{N}\right) & =0, \\
a \theta\left(v_{N}-v_{N-1}\right)-a\left(v_{N}-2 v_{N-1}+v_{N-2}\right)-a\left(v_{N}-v_{N-1}\right) & =\theta g_{1} .
\end{aligned}
$$

The equations (13)-(16) are obtained by considering the finite difference approximation at grid point $0,1, N-1$ and $N$, respectively. At the first and last grid point, SAT's have been applied.

It can be shown that (13)-(16) uniquely determine the coefficients $\sigma_{1-4}$. In fact, the coefficients are determined uniquely as long as well-posed boundary conditions and stability are guaranteed. If (13)-(16) had several solutions, the numerical solution $v$ would not be unique. Since stability implies convergence to the analytic solution, the continuous problem would also have several solutions, which contradicts well-posedness. This is shown in [11].

In this paper, we are not interested in the exact values of $\sigma_{1-4}$, and will instead use (14)-(15) to determine the order of magnitudes of $\sigma_{3,4}$. Inserting 
143

(12) into (14) yields

$$
\sum_{k=1}^{4}\left(\theta\left(K_{k}^{2}-1\right)-\frac{a}{2}\left(K_{k}-1\right)^{2}\left(K_{k}+\frac{1}{2}\right)\right) \sigma_{k}=\sum_{k=1}^{4} \tau_{k} \sigma_{k}=0
$$

where

$$
\tau_{k}=a \theta\left(K_{k}^{2}-1\right)-\frac{a}{2}\left(K_{k}-1\right)^{2}\left(K_{k}+\frac{1}{2}\right) .
$$

Since we are only interested in the order of magnitude, it suffice to consider the order of magnitudes of the $\tau_{k}$ 's. By using $K_{k}$, given by (11), in (18) one can conclude that

$$
\tau_{1}=0, \quad \tau_{2}=\mathcal{O}\left(h^{2}\right), \quad \tau_{3}=a, \quad \tau_{4}=a-2 a \theta+\mathcal{O}\left(h^{2}\right) .
$$

Inserting this result in (17) yields

$$
a \sigma_{3}+a\left(1-3 \theta+\mathcal{O}\left(h^{2}\right)\right) \sigma_{4}=-\tau_{2} \sigma_{2}=\mathcal{O}\left(h^{2}\right),
$$

if $\sigma_{2}$ is bounded, which is necessary for a stable scheme. Inserting (12) into (15) yields

$$
\sum_{k=1}^{4}\left(\theta\left(K_{k}^{2}-1\right) K_{k}^{N-2}-\frac{a}{2} K_{k}^{N-3}\left(K_{k}-1\right)^{2}\left(K_{k}+\frac{1}{2}\right)\right) \sigma_{k}=\sum_{k=1}^{4} \kappa_{k} \sigma_{k}=0
$$

where

With the values of $K_{k}$, given by (11), inserted into (20) for large values of $N$, one obtains

$$
a(-1)^{N-2} \sigma_{3}+a e^{-a / \epsilon}(-1)^{N-2}\left(1-2 \theta+\mathcal{O}\left(h^{2}\right)\right) \sigma_{4}=-\kappa_{2} \sigma_{2}=\mathcal{O}\left(h^{2}\right)
$$

where the approximation $\left|K_{4}\right|^{N}=e^{-a / \epsilon}+\mathcal{O}\left(h^{2}\right)$ is used and

$$
\kappa_{2}=a \theta\left(K_{2}^{2}-1\right) K_{2}^{N-2}-\frac{a K_{2}^{N-3}}{2}\left(K_{2}-1\right)^{2}\left(K_{2}+\frac{1}{2}\right)=\mathcal{O}\left(h^{2}\right) .
$$

Equations (19) and (21) can be written as

$$
\left[\begin{array}{cc}
a & a-3 a \theta+\mathcal{O}\left(h^{2}\right) \\
a(-1)^{N-2} & a e^{-a / \epsilon}(-1)^{N-2}(1+2 \theta)+\mathcal{O}\left(h^{2}\right)
\end{array}\right]\left[\begin{array}{l}
\sigma_{3} \\
\sigma_{4}
\end{array}\right]=\mathcal{O}\left(h^{2}\right) .
$$


It is easy to show that the matrix in (22) is non-singular for any $h$. Therefore, the coefficients $\sigma_{3,4}$ must be of order $\mathcal{O}\left(h^{2}\right)$. Hence, the oscillatory modes only contribute with second order terms.

We summarize the conclusions obtained so far in the following proposition.

Proposition 3. For the second order semi-discrete approximation (8), the spurious modes in (12) contribute with $\mathcal{O}\left(h^{2}\right)$ terms.

Remark. Neither the boundary terms nor the penalty parameters enter the derivation above, which means that the spurious part contributes with $\mathcal{O}\left(h^{2}\right)$ terms for all stable boundary procedures. Note also that if $\sigma_{2}=0$, equations (19) and (21) demand that both $\sigma_{3}$ and $\sigma_{4}$ must be zero, which shows that constant solutions are approximated exactly.

\section{Generalization to higher order schemes}

In the previous section, we demonstrated how a stable boundary procedure automatically makes the spurious modes vanish when second order SBP operators are used. A similar phenomena is actually valid for any stable high order finite difference scheme, as we will show in this section.

To ease the derivations, we restrict ourselves to diagonal matrices $P$. The following propositions and theorem are also true for a general symmetric matrix $P$, but the treatment of such operators will obscure the main points of the derivations. We start by stating

Proposition 4. Consider the approximation (8) with a $S B P$ operator $P^{-1} Q$ of internal order $2 m$ and a diagonal matrix $P$. The ansatz

$$
v_{i}(h) \sim K^{i}(h)
$$

where $h$ is the grid spacing and $i$ the grid point index, inserted into (8) leads to

$$
\lim _{h \rightarrow 0} K_{j}(h)=K_{j 0} .
$$

That is, all $K_{j}, j=1, \ldots, 4 m$, approach finite values as $h \rightarrow 0$.

Proof. Let $D=P^{-1} Q$ be a finite difference operator of order $2 m$ in the internal domain that approximates the first and second derivatives as $v_{x} \approx$ 
$D v, v_{x x} \approx D^{2} v$. A SBP finite difference operator of internal order $2 m$ uses the grid points $i-m, \ldots i+m$ to approximate the derivative at $i$, so we have

$$
(D v)_{i}=\left(P^{-1} Q v\right)_{i}=\frac{1}{h} \sum_{j=i-m}^{i+m} Q_{i, j} v_{j}=\frac{1}{h} \sum_{j=i-m}^{i+m} Q_{i, j} K^{j}
$$

$$
=\frac{1}{h}\left(\sum_{j=0}^{2 m} Q_{i, j+i-m} K^{j}\right) K^{i-m}=\frac{1}{h} \mathcal{P}_{2 m}(K) K^{i-m},
$$

where $\mathcal{P}_{2 m}(K)=\sum_{j=0}^{2 m} Q_{i, j+i-m} K^{j}$ is a polynomial of order $2 m$, independent of $h$. In (24), $Q$ is the matrix with the SBP-property in (8) and the index $i$ denotes an internal grid point. For example, in the fourth order case, we have

$$
(D v)_{i}=\frac{1}{h}\left(\frac{1}{12} v_{i-2}-\frac{2}{3} v_{i-1}+\frac{2}{3} v_{i+1}-\frac{1}{12} v_{i+2}\right) \Rightarrow
$$

$$
\frac{1}{h} P_{4}(K) K^{i-2}=\frac{1}{h}\left(\frac{1}{12}-\frac{2}{3} K+\frac{2}{3} K^{3}-\frac{1}{12} K^{4}\right) K^{i-2} .
$$

Similarly, for the second derivative approximation, one obtains

$$
\left(D^{2} v\right)_{i}=D \frac{1}{h} \mathcal{P}_{2 m}(K) K^{i-m}=\frac{1}{h^{2}} \mathcal{P}_{2 m}^{2}(K) K^{i-2 m} .
$$

We now use the results above to solve for the modes $K$ in (8). Inserting (24) and (25) into (8) at an internal grid point yields

$$
a(D v)_{i}-\epsilon\left(D^{2} v\right)_{i}=\frac{a}{h} \mathcal{P}_{2 m}(K) K^{i-m}-\frac{\epsilon}{h^{2}} \mathcal{P}_{2 m}^{2}(K) K^{i-2 m}=0 .
$$

By multiplying both sides of (26) with $h^{2} K^{m} / \epsilon$, one arrives at

$$
\mathcal{P}_{2 m}(K)\left(\mathcal{P}_{2 m}(K)-K^{m} \theta\right) K^{i-m}=0 .
$$

Note that $(10)$ is a special case of $(27)$, where $P_{2}(K)=\left(K^{2}-1\right) / 2$. According to (27), all $4 m$ modes are determined by

$$
\begin{aligned}
& \mathcal{P}_{2 m}(K)=0 \\
& \mathcal{P}_{2 m}(K)=\theta K^{m} .
\end{aligned}
$$

Note that (28) has $2 m$ solutions, all independent of $h$, that is $K_{j}=K_{0 j}=$ constant for $j=1, \ldots, 2 m$. 
Equation (29) is similar to (28), with a perturbation of magnitude $h$ at the right hand side. Since $\mathcal{P}_{2 m}$ is a polynomial, the solutions to (29) must therefore be close to the ones of (28), that is $K_{j p}=K_{j 0}+\mathcal{O}(h)$ where the index $p$ denotes the perturbed solutions to (29). Hence, for any solution to (27), we have $\lim _{h \rightarrow 0} K_{j}(h)=K_{j 0}=$ constant.

With all modes obtained from (27), the final form of the solution is

$$
v_{k}=\sum_{j} \sigma_{j} K_{j}^{k}
$$

where the modes $K$ are the solutions to (27) and $\sigma_{j}$ are constants to be determined by the boundary conditions. We state the following lemma and proposition.

Lemma 1. Let the numerical solution at grid point $k$ be given by (30), such that the solution at every grid point can be written $v=\hat{K} \tilde{\sigma}$, where $\tilde{\sigma}$ is a $4 m \times 1$ vector containing the coefficients $\sigma_{j}$ and $\hat{K}$ is a $N \times 4$ m matrix with $\hat{K}_{i j}=K_{j}^{i-1}$, where all $K_{j}$ are distinct. The rank of $\hat{K}$ is then $4 m$ and, consequently, $\hat{K}^{*} \hat{K}$ is non-singular.

\section{Proof. See Appendix A.}

Proposition 5. Let the numerical solution to (8) be given by (30) where $k$ denotes the grid point and the modes $K_{j}$ are distinct. If $v$ approximates the analytic solution, $u$, with an accuracy $\mathcal{O}\left(h^{m+1}\right)$, then all $\sigma_{j}$ converges to fixed values.

Proof. The vector $v$, which represents the solution at every grid point, can be written as

$$
v=\hat{K} \tilde{\sigma},
$$

where $v, \tilde{\sigma}$ and $\hat{K}$ are the vectors and matrices defined in Lemma 1 . If the overall accuracy of the scheme is $\mathcal{O}\left(h^{m+1}\right)$, the numerical solution approximates the analytic one, $u$, as

$$
v-u=\hat{K} \tilde{\sigma}-u=\mathcal{O}\left(h^{m+1}\right) .
$$

By multiplying (32) with $\left(\hat{K}^{*} \hat{K}\right)^{-1} \hat{K}^{*}$ from the left, one obtains the coefficients $\tilde{\sigma}$,

$$
\tilde{\sigma}=\left(\hat{K}^{*} \hat{K}\right)^{-1} \hat{K}^{*} u+\mathcal{O}\left(h^{m+1}\right),
$$


and we observe that $\lim _{h \rightarrow 0} \tilde{\sigma}=\left(\hat{K}^{*} \hat{K}\right)^{-1} \hat{K}^{*} u$. Since the matrix $\left(\hat{K}^{*} \hat{K}\right)^{-1}$ is non-singular (as stated in Lemma 1 ) and converges to a constant matrix, $\tilde{\sigma}$ is uniquely determined by $\hat{K}^{*} u$, which means that every $\sigma_{j}$ converges towards fixed values according to (33).

Remark. It can be shown that (28) has distinct roots for all SBP schemes with up to, at least, eight order accuracy in the interior. Proposition 5 is therefore valid for all such schemes.

The numerical solution at any grid point $k$ can be divided into positive and negative (spurious) modes, such that

$$
v_{k}=\left(v_{k}\right)_{+}+\left(v_{k}\right)_{-}=\sum_{j_{+}} \sigma_{j+} K_{j+}^{k}+\sum_{j_{-}} \sigma_{j-} K_{j-}^{k} .
$$

In (34), the spurious modes are denoted by $K_{j-}<0$ and the converging modes by $K_{j+}>0$. We are now ready to state the main result of this paper.

Theorem 1. If $v=v_{+}+v_{-}$in (34) is approximated with the $m+1$ order accurate scheme (8) and $u(x)$ is the analytic solution to (1) in the steadystate limit, then

$$
v_{+}-u(x)=\mathcal{O}\left(h^{m+1}\right), \quad v_{-}=\mathcal{O}\left(h^{m+1}\right) .
$$

Proof. Assuming that well-posed boundary conditions and a stable and accurate numerical approximation exist, then

$$
\|v-u\|_{P}^{2}=\left\|v_{+}-u\right\|_{P}^{2}+\left\|v_{-}\right\|_{P}^{2}+2 v_{-}^{T} P\left(v_{+}-u\right)=\mathcal{O}\left(h^{2 m+2}\right),
$$

since the overall accuracy of the scheme is $\|v-u\|_{P}=\mathcal{O}\left(h^{m+1}\right)$.

For (35) to be valid, the left hand side needs to be of the same order of magnitude as the right-hand side. From Proposition 4 and 5 we know that $v_{+}$approaches a function $\hat{v}_{+}(x)$, and therefore

$$
\lim _{h \rightarrow 0}\left\|v_{+}(h)\right\|_{P}^{2}=\int_{0}^{1} \hat{v}_{+}^{2} d x,
$$

since both $\sigma_{j+}(h)$ and $K_{j+}(h)>0$ approach finite values and the weight matrix $P$ is a discrete integration operator of order $m+1$ [12]. Similarly, the norm of the spurious part also approaches some finite value,

$$
\lim _{h \rightarrow 0}\left\|v_{-}(h)\right\|_{P}^{2}=\int_{0}^{1} \hat{v}_{-}^{2} d x .
$$


Equation (35) can be written as

$$
v_{-}^{T} P\left(v_{+}-u\right)=-\frac{\left\|v_{+}-u\right\|_{P}^{2}+\left\|v_{-}\right\|_{P}^{2}}{2}+\mathcal{O}\left(h^{2 m+2}\right) .
$$

The left-hand side of (36) can be written as

$$
\begin{aligned}
v_{-}^{T} P\left(v_{+}-u\right)= & \sum_{i=0}^{N} P_{i i}\left(v_{-}\right)_{i}\left(v_{+}-u\right)_{i}=\sum_{i=0}^{N} \sum_{j_{-}} P_{i i}\left(v_{+}-u\right)_{i} \sigma_{j-} K_{j-}^{i}= \\
& \sum_{i=0}^{N} P_{i i} \sigma_{1-} K_{1-}^{i}\left(v_{+}-u\right)_{i}+\sum_{i=0}^{N} P_{i i} \sigma_{2-} K_{2-}^{i}\left(v_{+}-u\right)_{i}+\ldots,
\end{aligned}
$$

where we have used $\left(v_{-}\right)_{i}=\sum_{j_{-}} \sigma_{j-} K_{j-}^{i}$. All $K_{j_{-}}$are negative and converge to fixed values. For sufficiently small $h$, a limit-value of (37) does not exist unless $\sigma_{j-} \rightarrow 0$ or $\left(v_{+}-u\right)_{i} \rightarrow 0$. We can thus conclude that both of the norms $\left\|v_{+}-u\right\|_{P}$ and $\left\|v_{-}\right\|_{P}$ must approach zero during mesh refinement, since (36) and (37) would yield a contradiction otherwise. If none of them converge, (36) can not be satisfied since the limit on the left-hand side does not exist; if only one of the norms converges, the limit on the left-hand side exists and is zero, which is then inconsistent with the right-hand side. Hence, both norms must approach zero with a rate $\mathcal{O}\left(h^{m+1}\right)$.

Next, we exemplify the theoretical results in the second order case, where all details are known. First, note that two of the solutions to (10), given by $(11)$, are constant $( \pm 1)$ and the other two are identical, with a perturbation $\mathcal{O}(h)$. Hence, Proposition 4 is, at least, true for the second order case. To determine the coefficients $\sigma_{j}$, we need the analytic solution $u(x)$. In our case, we can easily invent one; $u(x)=1+e^{a / \epsilon x}$ satisfies (1) in the steady-state limit and with the boundary data chosen accordingly. In our test-case, $a=1$ and $\epsilon=0.1$. The numerical solution is identical to the analytic one if $\sigma_{1,2}=1$ and $\sigma_{3,4}=0$ in the limit $N \rightarrow \infty$. According to Proposition 5, we have

$$
\tilde{\sigma}=\left(\hat{K}^{*} \hat{K}\right)^{-1} \hat{K}^{*} u+\mathcal{O}\left(h^{m+1}\right)
$$

where $u$ is the exact solution injected at the grid points and the vector $\tilde{\sigma}=$ $\left[\sigma_{1}, \sigma_{2}, \sigma_{3}, \sigma_{4}\right]^{T}$. Equation (38) implies that $\left(\hat{K}^{*} \hat{K}\right)^{-1} \hat{K}^{*} u \rightarrow \tilde{\sigma}_{0}=[1,1,0,0]^{T}$, which are the coefficients for the exact solution. In Table 1, we show how 
Table 1: Rate of convergence of the coefficients $\sigma_{j}$ during mesh-refinement.

\begin{tabular}{c|c|c} 
Number of grid points & $\left\|\left(\hat{K}^{*} \hat{K}\right)^{-1} \hat{K}^{*} u-\tilde{\sigma}_{0}\right\|_{2}$ & Rate of convergence \\
\hline 10 & $3.8 \cdot 10^{-3}$ & - \\
20 & $9.6 \cdot 10^{-4}$ & 1.98 \\
40 & $2.4 \cdot 10^{-4}$ & 2.00 \\
80 & $6.0 \cdot 10^{-5}$ & 2.00 \\
160 & $1.5 \cdot 10^{-5}$ & 2.00
\end{tabular}

$\left(\hat{K}^{*} \hat{K}\right)^{-1} \hat{K}^{*} u$ converges to $\tilde{\sigma}_{0}$ during mesh-refinement, thus showing the validity of Proposition 5. The rate of convergence is two, as Theorem 1 indicates. We can then conclude that

$$
\left\|v_{-}\right\|_{P}=\left|\sigma_{3} \sum_{i} P_{i, i} K_{3}^{i}+\sigma_{4} \sum_{i} P_{i, i} K_{4}^{i}\right|=\mathcal{O}\left(h^{2}\right)
$$

and

$$
\left\|v_{+}-u\right\|_{P}=\sqrt{\sum_{i} P_{i i}\left[\left(\sigma_{1}-1\right)+\left(\sigma_{2} K_{2}^{i}-e^{a / \epsilon x}\right)\right]^{2}}=\mathcal{O}\left(h^{2}\right),
$$

since $K_{2}^{i}=e^{a / \epsilon x}+\mathcal{O}\left(h^{2}\right)$. This exemplifies the validity of Proposition 4, Proposition 5 and Theorem 1 for the second order scheme.

\section{Numerical results}

We exemplify the theoretical results in one and two space dimensions.

\subsection{One dimensional experiments}

Numerical experiments have been performed on problem (5) with $a=1$, $\epsilon=0.1$, the boundary data $g_{0}=1, g_{1}=-1$ and the initial condition $f(x)=0$. The exact solution is then $u(x)=1-\exp (10(x-1))$. The $S B P(2,1)$ scheme (a scheme with second order accuracy in the interior and first order at the boundaries) is integrated in time using the classical explicit Runge-Kutta of fourth order accuracy until the system have reached the steady-state solution. The domain considered is $x \in[0,1]$.

Figure 1-3 show how the oscillations vanish as the grid is refined. The rate of convergence and error levels are displayed for $\epsilon=0.1$ in Table 2-4, and one can see that the rates of convergence approach the design order of the 


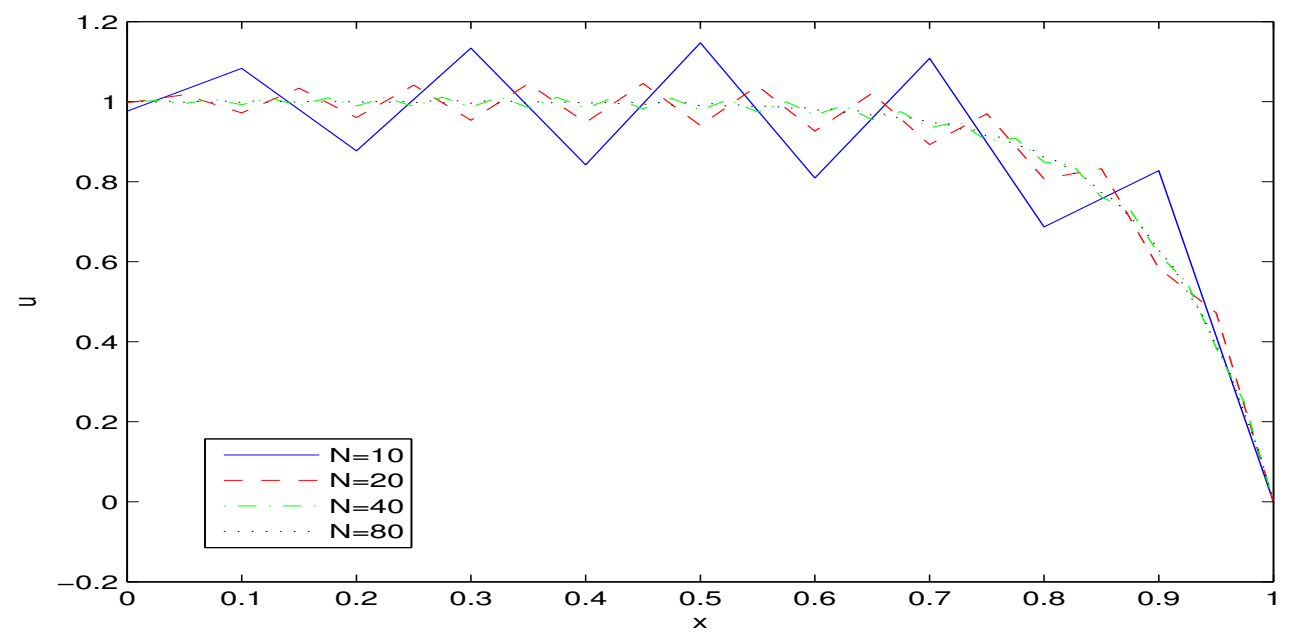

Figure 1: The solution as a function of $x$ at steady-state for different meshes. An $S B P(2,1)$ scheme is used and $a=1, \epsilon=0.1$.

scheme. Moreover, increasing the order of accuracy of the scheme drastically reduce the errors from the spurious modes.

As one can see in Figure 1-3, the oscillations are only problematic if one uses a coarse mesh and scheme of low order. When increasing the order of the scheme or the number of grid points, the oscillations vanish rapidly. Consequently, using wide stencils does not cause any real problems.

\subsection{Two dimensional experiments}

To exemplify that the one-dimensional analysis hold also in multiple di-

Table 2: Error and convergence rate for different meshes and $a=1, \epsilon=0.1$. An $S B P(2,1)$ scheme is used.

\begin{tabular}{c|c|c} 
Number of grid points & Error at steady-state & Rate of convergence \\
\hline 10 & 0.20 & - \\
20 & 0.08 & 1.32 \\
40 & 0.025 & 1.68 \\
80 & 0.007 & 1.84 \\
160 & 0.002 & 1.81 \\
320 & 0.00051 & 1.99
\end{tabular}




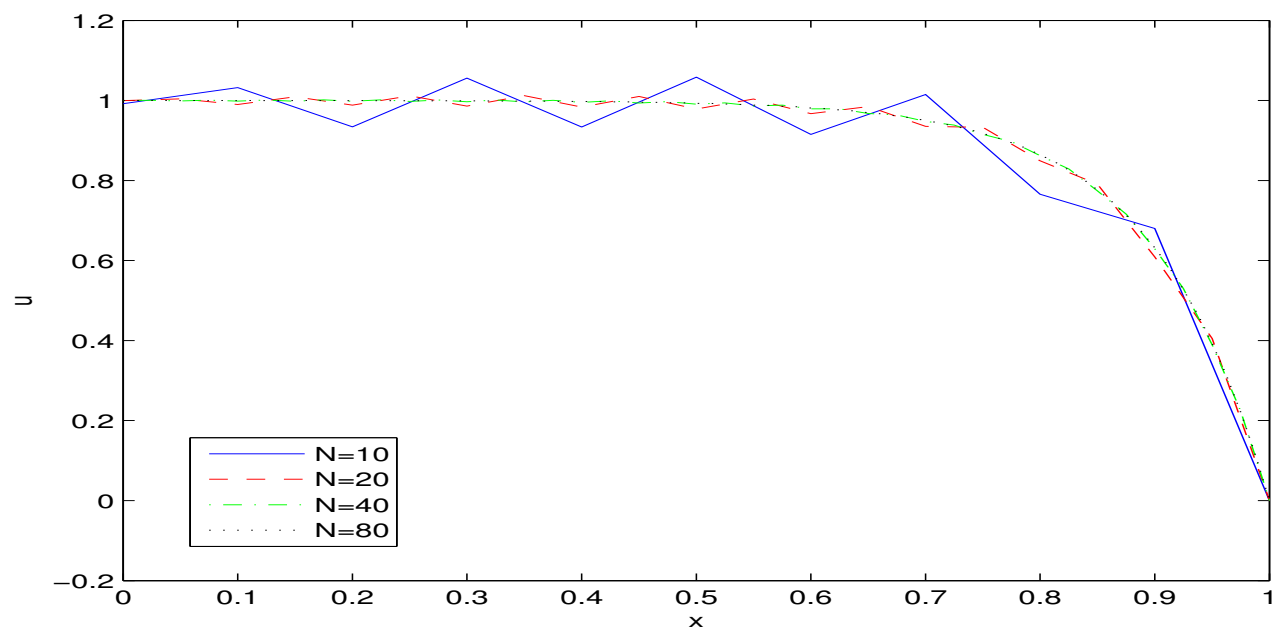

Figure 2: The solution as a function of $x$ at steady-state for different meshes. An $S B P(4,2)$ scheme is used and $a=1, \epsilon=0.1$.

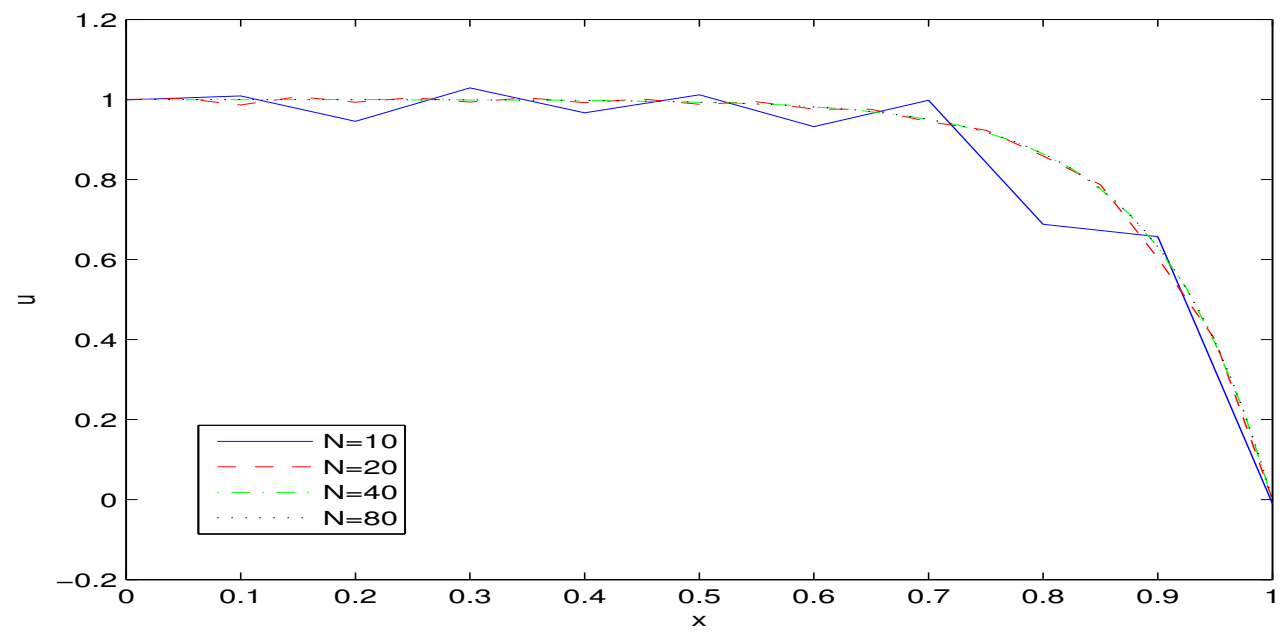

Figure 3: The solution as a function of $x$ at steady-state for different meshes. An $S B P(6,3)$ scheme is used and $a=1, \epsilon=0.1$. 
Table 3: Error and convergence rate for different meshes and $a=1, \epsilon=0.1$. An $S B P(4,2)$ scheme is used.

\begin{tabular}{c|c|c} 
Number of grid points & Error at steady-state & Rate of convergence \\
\hline 20 & $1.3 \times 10^{-2}$ & - \\
40 & $2.3 \times 10^{-3}$ & 2.5 \\
80 & $3.3 \times 10^{-4}$ & 2.8 \\
160 & $4.4 \times 10^{-5}$ & 2.9 \\
320 & $5.7 \times 10^{-6}$ & 2.9
\end{tabular}

Table 4: Error and convergence rate for different meshes and $a=1, \epsilon=0.1$. An $S B P(6,3)$ scheme is used.

\begin{tabular}{c|c|c} 
Number of grid points & Error at steady-state & Rate of convergence \\
\hline 20 & $7.9 \times 10^{-3}$ & - \\
40 & $6.2 \times 10^{-4}$ & 3.7 \\
80 & $3.5 \times 10^{-5}$ & 4.1 \\
160 & $1.8 \times 10^{-6}$ & 4.3 \\
320 & $8.8 \times 10^{-8}$ & 4.4
\end{tabular}

mensions, consider the problem,

$$
\begin{aligned}
u_{t}+u_{x}+u_{y} & =\epsilon\left(u_{x x}+u_{y y}\right), \quad x, y \in[0,1], t \geq 0 \\
u(0, y, t)-\epsilon u_{x}(0, y, t) & =g_{0, x}(y, t), \quad y \in[0,1], t \geq 0 \\
\epsilon u_{x}(1, y, t) & =g_{1, x}(y, t), \quad y \in[0,1], t \geq 0 \\
u(x, 0, t)-\epsilon u_{y}(x, 0, t) & =g_{0, y}(x, t), \quad x \in[0,1], t \geq 0 \\
\epsilon u_{y}(x, 1, t) & =g_{1, y}(x, t), \quad x \in[0,1], t \geq 0 \\
u(x, y, 0) & =f(x, y) .
\end{aligned}
$$

We choose the initial data $f(x, y)=0, \epsilon=0.1$, the steady state solution to be $u_{S S}=1-\exp ((x+y-2) / \epsilon)$ and the boundary data accordingly. An $\operatorname{SBP}(4,2)$ operator is used for the spatial discretization and a fourth order Runge-Kutta method is used to integrate in time until the system have reached steady state.

In Figure 4-6, the solution at steady state is displayed when using $N=10,20,40$ grid points in each space direction. The spurious modes can best be seen close to the line $x=1$, and we observe that they vanish during 


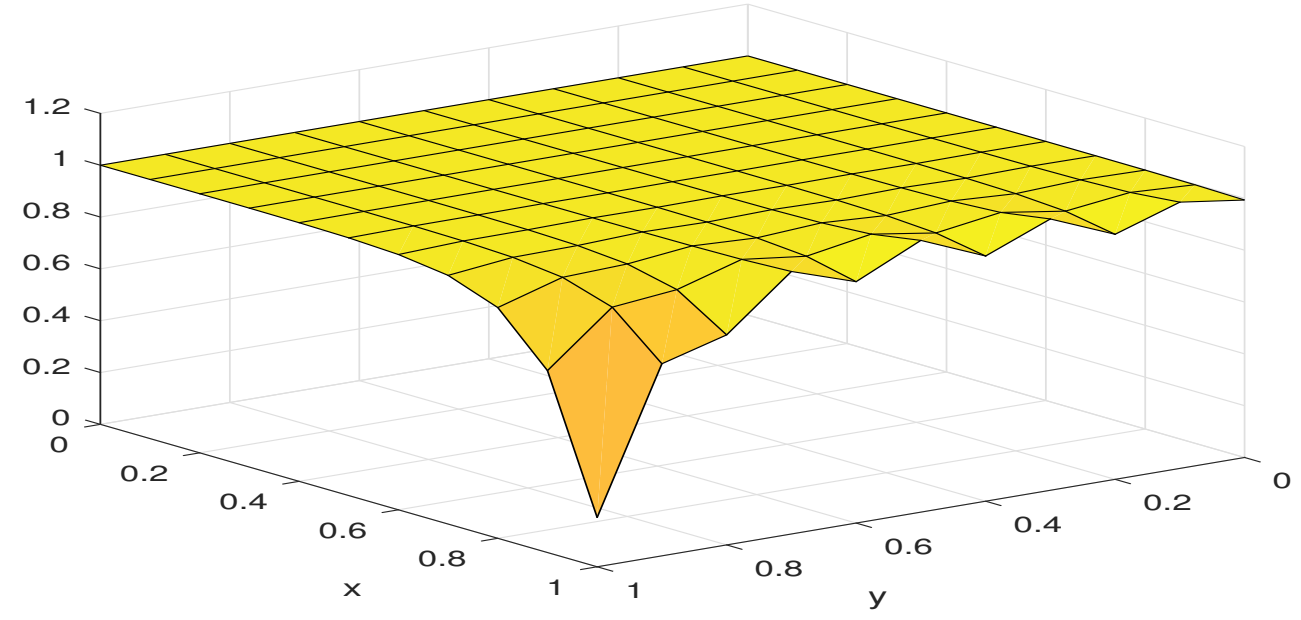

Figure 4: The solution to (39) at steady state when using $N=10$ points in each space direction.

mesh refinement, in the same way as for the one-dimensional case. It can also be shown that the convergence is similar.

\section{Conclusions}

We have shown that the spurious modes, for the advection-diffusion equation, are of the same order of magnitude as the truncation error when using wide operators for approximating the second derivative. The theoretical results are consistent with the numerical experiments.

The results derived in this work are independent of the boundary procedure or the numerical scheme. Consequently, the spurious modes vanish with a rate equal to the order of the scheme for any numerical method as long as it is stable.

As the spurious modes only contribute with errors of the same magnitude as the order of the scheme, the oscillations can always be removed by choosing a scheme of high order or using a fine mesh. Since the oscillations converge with the same rate as the scheme, no real problems exist for wide operators. 


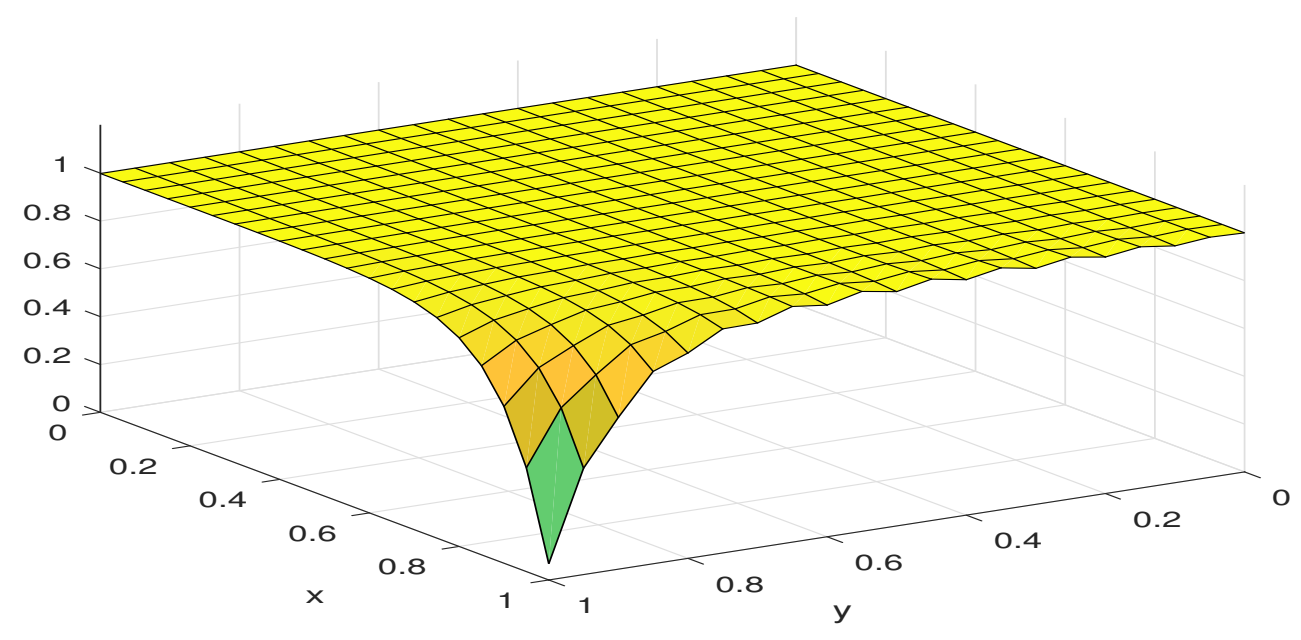

Figure 5: The solution to (39) at steady state when using $N=20$ points in each space direction.

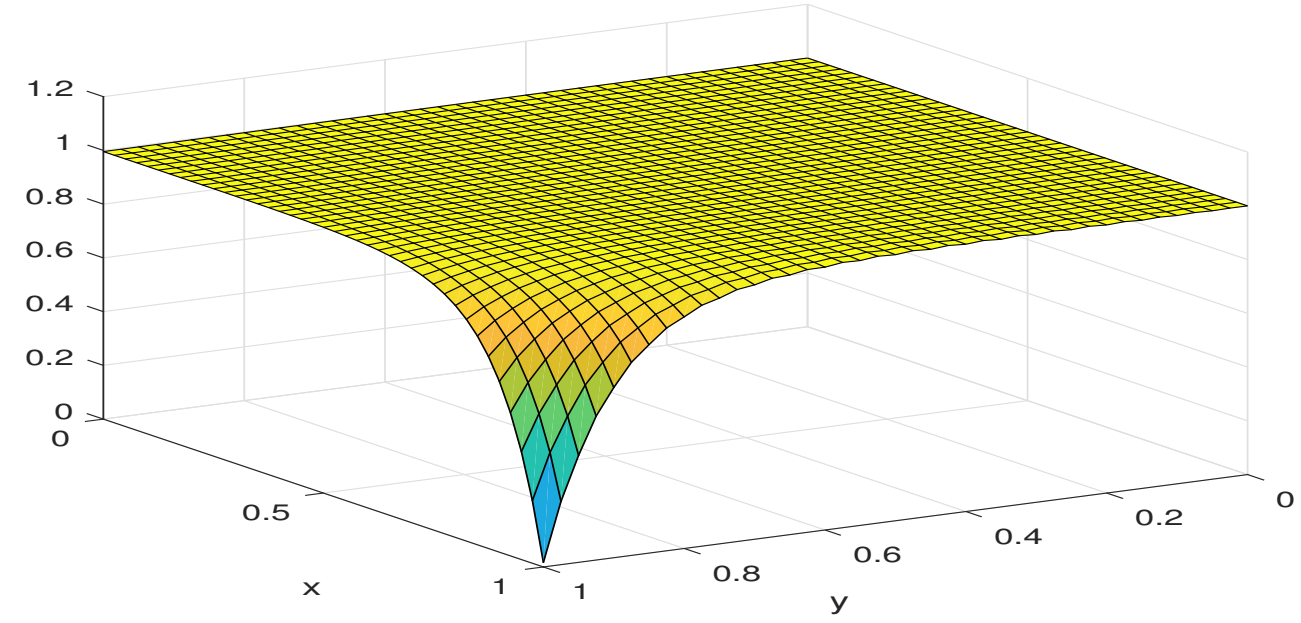

Figure 6: The solution to (39) at steady state when using $N=40$ points in each space direction. 


\section{Acknowledgements}

This project was funded by the Swedish e-science Research Center (SeRC). The funding source had no involvement in the study design, collection and analysis of data, or in writing and submitting this article.

\section{Appendix A}

We will in this appendix prove that the $N-b y-4 m$ matrix $\hat{K}$ in (31) has rank $4 m$ and, consequently, $\hat{K}^{*} \hat{K}$ is non-singular. First, we note that there are $2 m$ solutions to (28) and $2 m$ similar solutions to (29) with a perturbation $\mathcal{O}(h)$. It can be shown that the $2 m$ solutions to (28) are distinct for all SBP operators with up to eighth order internal accuracy; consequently, all solutions to (28) and (29) are distinct with a perturbation of at least $\mathcal{O}(h)$. We have

$$
\hat{K}=\left[\begin{array}{cccc}
1 & 1 & \cdots & 1 \\
K_{1} & K_{2} & \cdots & K_{4 m} \\
K_{1}^{2} & K_{2}^{2} & \cdots & K_{4 m}^{2} \\
\vdots & \vdots & \vdots & \vdots \\
K_{1}^{N} & K_{2}^{N} & \cdots & K_{4 m}^{N}
\end{array}\right] .
$$

The matrix $\hat{K}$ has rank $4 m$ if one can find $4 m$ linearly independent rowvectors.

We choose $4 m$ rows from $\hat{K}$ and put them as column vectors in a matrix that we call $\tilde{K}$. As first column, we choose the row vector $\hat{K}_{1}=$ $\left[K_{1}^{N / 4 m}, K_{2}^{N / 4 m}, \cdots, K_{4 m}^{N / 4 m}\right]$, the second as $\hat{K}_{2}=\left[K_{1}^{2 N / 4 m}, K_{2}^{2 N / 4 m}, \cdots, K_{4 m}^{2 N / 4 m}\right]$ and so forth. Then, we have

$$
\tilde{K}=\left[\begin{array}{cccc}
\tilde{K}_{1} & \tilde{K}_{1}^{2} & \cdots & \tilde{K}_{1}^{4 m} \\
\tilde{K}_{2} & \tilde{K}_{2}^{2} & \cdots & \tilde{K}_{2}^{4 m} \\
\vdots & \vdots & \vdots & \vdots \\
\tilde{K}_{4 m} & \tilde{K}_{4 m}^{2} & \cdots & \tilde{K}_{4 m}^{4 m}
\end{array}\right],
$$

where $\tilde{K}_{j}=K_{j}^{N / 4 m}$. If these $4 m$ rows are linearly independent, i.e $\tilde{K}$ is non-singular, then the matrix $\hat{K}$ has rank $4 m$.

Remark. In (A-1), we have chosen $\tilde{K}_{i}=K_{i}^{N / 4 m}$ for simplicity. However, one can also choose $\tilde{K}_{i}=K_{i}^{N / k}$ for any integer $N>>>1$, and the following arguments would still be valid. 
We note that $(\mathrm{A}-1)$ is a $4 m-b y-4 m$ Vandermonde matrix and its determinant is

$$
\operatorname{det}(\tilde{K})=\prod_{i} \tilde{K}_{i} \prod_{1 \leq i<j \leq 4 m}\left(\tilde{K}_{j}-\tilde{K}_{i}\right)
$$

where $\tilde{K}_{i}=K_{i}^{N / 4 m}$. Note that if all $\tilde{K}_{i}$ in (A-1) are distinct and does not approach each other as $N \rightarrow \infty$, then $\tilde{K}$ is non-singular. By Proposition 4, there exist $2 m$ constant $K$ and $2 m$ identical $K$ 's with a perturbation $\mathcal{O}(h)$. We denote these modes as $K_{c}$ and $K_{p}$ according to

$$
\begin{aligned}
& K_{c}=K_{0} \\
& K_{p}=K_{0}+\Delta K h+\mathcal{O}\left(h^{2}\right)
\end{aligned}
$$

where $\Delta K \neq 0$. For large $N$, we have

$$
\tilde{K}_{p}=K_{p}^{N / 4 m} \approx\left(K_{0}+\Delta K h\right)^{N / 4 m} \approx K_{0}^{N / 4 m} e^{4 m \Delta K / K_{0}} \neq \tilde{K}_{c}
$$

and all $\tilde{K}$ are thus distinct in $(\mathrm{A}-1)$. Consequently, $\tilde{K}$ is non-singular. For the second order accurate scheme, $K_{c}= \pm 1$, and all $K_{j}$ are therefore distinct for all $h$. One can therefore conclude that Lemma 1 is true for the second order scheme.

For higher order schemes, however, there exist modes that vanish inside the domain, that is $\left|K_{j}\right|^{i} \rightarrow 0$ for some index $j$ and grid points $i$. In this case, $\tilde{K}$ will be singular (some rows will contain only zeros), and we need to choose other rows from $\hat{K}$ to form a linearly independent set of dimension $4 \mathrm{~m}$. First, we note that the vanishing modes always occur in pairs: one which is constant, and another one with a perturbation $\mathcal{O}(h)$. For the moment, let us assume that there exist two vanishing modes, which is the case for the SBP 42 scheme, and denote them as $K_{1,2}$. The structure of the matrix $\hat{K}$ is then

$$
\hat{K}=\left[\begin{array}{cc}
V & W \\
\delta V & A
\end{array}\right]
$$

where the matrix

$$
A=\left[\begin{array}{cccc}
K_{3}^{N / 4 m-2} & K_{4}^{N / 4 m-2} & \cdots & K_{4 m}^{N / 4 m-2} \\
K_{3}^{N / 4 m-1} & K_{4}^{N / 4 m-1} & \cdots & K_{4 m}^{N / 4 m-1} \\
\vdots & \vdots & \vdots & \vdots \\
K_{3}^{N} & K_{4}^{N} & \cdots & K_{4 m}^{N}
\end{array}\right]
$$


380

381

382

$$
V=\left[\begin{array}{cc}
1 & 1 \\
K_{1} & K_{2} \\
\vdots & \vdots \\
K_{1}^{N / 4 m-3} & K_{2}^{N / 4 m-3}
\end{array}\right]
$$

$$
W=\left[\begin{array}{ccc}
1 & \cdots & 1 \\
K_{3} & \cdots & K_{4 m} \\
\vdots & \vdots & \vdots \\
K_{3}^{N / 4 m-3} & \cdots & K_{4 m}^{N / 4 m-3}
\end{array}\right] \text {, }
$$

and

$$
\delta V=\left[\begin{array}{cc}
K_{1}^{N / 4 m-2} & K_{2}^{N / 4 m-2} \\
\vdots & \vdots \\
K_{1}^{N} & K_{2}^{N}
\end{array}\right] \approx 0
$$

since $\left|K_{1,2}\right|<0$ and $N$ is large. The matrix $A$ has full rank since none of the modes $K_{3 \cdots 4 m}$ vanish, as concluded earlier in this section. Consequently, $\hat{K}$ will have full rank if the column vectors of $V$ are linearly independent, since we, in that case, have found $4 m$ linearly independent column vectors. We know that $K_{2}=K_{1}+\Delta K h+\mathcal{O}\left(h^{2}\right)$ where $\Delta K \neq 0$, so

$$
V \approx\left[\begin{array}{cc}
1 & 1 \\
K_{1} & K_{1}+\Delta K h \\
K_{1}^{2} & K_{1}^{2}\left(1+\frac{\Delta K}{K_{1}} h\right)^{2} \\
\vdots & \vdots \\
K_{1}^{N / 4 m-3} & K_{1}^{N / 4 m-3}\left(1+\frac{\Delta K}{K_{1}} h\right)^{N / 4 m-3}
\end{array}\right]
$$

by neglecting higher order terms. Since

$$
\lim _{k \rightarrow \infty}\left(1+\frac{\Delta K}{K_{1}} h\right)^{k}=e^{\frac{\Delta K}{K_{1}} k h} \neq 1,
$$

the column vectors are linearly independent and $\hat{K}$ has therefore full rank. Sylvester's rank inequality states that

$$
\operatorname{rank}(A B) \geq \operatorname{rank}(A)+\operatorname{rank}(B)-k,
$$


if $A$ is a $l-b y-k$ matrix and $B$ is $k-b y-n$ for any integers $l$ and $n$. Using $k=4 m, A=\hat{K}^{*}$ and $B=\hat{K}$, one arrives at

$$
\operatorname{rank}\left(\hat{K}^{*} \hat{K}\right) \geq \operatorname{rank}\left(\hat{K}^{*}\right)+\operatorname{rank}(\hat{K})-4 m=4 m+4 m-4 m=4 m
$$

and the matrix $\hat{K}^{*} \hat{K}$ must have rank $4 m$ and is therefore non-singular. This concludes the proof of Lemma 1.

Remark. If more than two vanishing modes exist, the procedure above is repeated by adding additional columns in the matrix $V$. The proof would therefore be similar.

[1] B. Engquist and A. Majda, Absorbing boundary conditions for the numerical simulation of waves, Mathematics of Computation 139, (1977), 629-651

[2] C.W Rowley and T. Colonius, Discretely non-reflecting boundary conditions for linear hyperbolic systems, Journal of Computational Physics 157, (2000), 500-583

[3] K. Mattsson and M. Svärd and J. Nordström, Stable and accurate artificial dissipation, Journal of Scientific Computing 21, (2004), 57-79

[4] J. Nordström, Conservative finite difference formulations, variable coefficients, energy estimates and artificial dissipation, Journal of Scientific Computing 29, (2005), 375-404

[5] M.Svärd and J. Nordström, Review of summation-by-parts schemes for initial-boundary-value problems, Journal of Computational Physics 268, (2014), 17-38

[6] K. Mattsson and J. Nordström, Summation by parts operators for finite difference approximations of second derivatives, Journal of Computational Physics 199, (2004), 503-540

[7] M. Carpenter and J. Nordström and D. Gottlieb, A stable and conservative interface treatment of arbitrary spatial accuracy, Journal of Computational Physics 148, (1999), 341-365

[8] B. Strand, Summation by parts for finite difference approximations for $\frac{d}{d x}$, Journal of Computational Physics 110, (1994), 47-67 
[9] M. Carpenter and D. Gottlieb and S. Abarbanel, Time-stable boundary conditions for finite-difference schemes solving hyperbolic systems: methodology and application to high-order compact schemes, Journal of Computational Physics 111, (1994), 220-236

[10] J. Nordström and M. Svärd, Well-posed boundary conditions for the Navier-Stokes equations, SIAM Journal of Numerical Analysis 43, (2013), 1231-1255

[11] M. Svärd and J. Nordström, On the order of accuracy for finite difference approximations of initial-boundary value problems, Journal of Computational Physics 218, (2006), 333-352

[12] J.E Hicken and D.W Zingg, Summation-by-parts and high-order quadrature, Journal of Computational and Applied Mathematics 237, (2013), $111-125$ 